\title{
The clinical spectrum of Blake's pouch cyst: report of six illustrative cases
}

\author{
Erwin M. J. Cornips • Geke M. Overvliet • Jacobiene W. Weber • Alida A. Postma • \\ Christianne M. Hoeberigs • Marcella M. L. L. Baldewijns • Johannes S. H. Vles
}

Received: 19 October 2009 / Accepted: 20 January 2010/Published online: 3 March 2010

(C) The Author(s) 2010. This article is published with open access at Springerlink.com

\begin{abstract}
Introduction Although Blake's pouch cyst (BPC) is frequently mentioned in the spectrum of posterior fossa cysts and cystlike malformations since its first description in 1996, its natural history, clinical presentation, specific imaging characteristics, optimal treatment, and outcome are relatively unknown. Consequently, BPC may still be underdiagnosed. We therefore report six cases ranging from a fatal hydrocephalus in a young boy, over an increasing head circumference with or without impaired neurological development in two infants, to a decompensating hydrocephalus at an advanced age.

Discussion We focus on their radiological uniformity, which should help making the correct diagnosis, and widely variable clinical presentation, which includes adult cases as well. Differentiating BPC from other posterior fossa cysts and cystlike malformations and recognizing the accompanying hydrocephalus are essentially noncommunicating, not only have important implications on clinical
\end{abstract}

Cornips and Overvliet contributed equally to the article.

E. M. J. Cornips $(\bowtie) \cdot$ G. M. Overvliet

Department of Neurosurgery,

Maastricht University Medical Center,

P.O. Box 5800, 6202 AZ Maastricht, The Netherlands

e-mail: e.cornips@mumc.nl

A. A. Postma $\cdot$ C. M. Hoeberigs

Department of Radiology, Maastricht University Medical Center, Maastricht, The Netherlands

M. M. L. L. Baldewijns

Department of Pathology, Maastricht University Medical Center,

Maastricht, The Netherlands

J. W. Weber · J. S. H. Vles

Department of Child Neurology,

Maastricht University Medical Center,

Maastricht, The Netherlands management but also on genetic counseling, which is unnecessary in case of BPC. In our experience, endoscopic third ventriculostomy is a safe and effective treatment option, avoiding the risks and added morbidity of open surgery, as well as many shunt-related problems.

Keywords Blake's pouch cyst · Dandy-Walker malformation · Hydrocephalus · Posterior fossa cyst .

Treatment

\section{Introduction}

Posterior fossa cysts and cystlike malformations are frequently observed on neuroimaging studies in children. These include retrocerebellar cysts, arachnoid cysts, and the Dandy-Walker complex (DWC), including the classic DandyWalker malformation, Dandy-Walker variant, megacisterna magna, and (either in the Dandy-Walker complex or as a separate entity) Blake's pouch cyst (BPC) $[1-4,6,7,10,11]$. From a clinical point of view, megacisterna magna and arachnoid cysts usually are incidental findings, whereas cysts in the DWC are associated with other developmental anomalies (cerebellar hemispheres and vermis) and most often present with hydrocephalus early in life $[1-4,6,7,10$, 11]. Within the radiological spectrum of posterior fossa cysts and cystlike malformations, BPC is a less well-known yet separate entity, although by some authors still classified in the DWC. Interestingly, BPC is capable of producing a broad spectrum of symptoms and signs ranging from asymptomatic to a full-blown hydrocephalus [6]. In this paper, we present six patients of different ages in whom we diagnosed a BPC in the past 4 years. We elaborate on their radiological uniformity on the one hand and widely variable clinical presentation on the other hand. 


\section{Case material}

Case 1

At the age of 1 month, an otherwise healthy boy started vomiting and refusing to drink and was admitted to the hospital 3 days thereafter. The next day, he started posturing, and over the following days, he became obtunded and ultimately developed an ophistotonus. At this point, he was sedated, intubated, and urgently transferred to our hospital. Upon arrival, we noted a pale skin, congested scalp veins, an extremely tense fontanel, markedly separated sutures, and slowly reactive pupils. Magnetic resonance (MR) imaging performed in the referring hospital demonstrated a marked tetraventricular hydrocephalus with bulging third ventricular floor, downward bending mammillary bodies, an open aqueduct, and apparent fourth ventricular outflow obstruction (Fig. 1a, b). The cerebellum was not rotated, and the overall picture was that of a BPC. We performed an emergency endoscopic third ventriculostomy (ETV) leaving an external ventricular drain to ensure adequate cerebrospinal fluid (CSF) drainage until clinical reevaluation. As expected, intracranial pressure was extremely high. As a complicating factor, coagulation status was suboptimal, which was later shown to be caused by vitamin K depletion due to biliary atresia. Postoperatively, the boy developed a lasting nonconvulsive status epilepticus with widespread cerebral ischemic lesions and hemorrhage in the right frontal surgical trajectory. After 14 days without any clinical improvement, supportive therapy was discontinued and the boy died. Postmortem examination confirmed the presence of a hemorrhagic BPC (Fig. 1d), massive cerebral ischemia, and biliary atresia of unknown origin.

\section{Case 2}

At the age of 2 years and 4 months, an otherwise healthy boy presented to our outpatient clinic with delayed gait development. He had started to walk at the age of 2 years and 2 months with a broad based gait, assisted by one of his
Fig. 1 a Preoperative sagittal T2-weighted MR image demonstrating severe hydrocephalus with bulging third ventricular floor, downward bending mammillary bodies, and an open aqueduct. The cerebellum is not rotated. A thin vertical line between dilated fourth ventricle and cisterna magna indicates a Blake's pouch cyst. b Preoperative axial T1-weighted MR image demonstrating a bilateral indentation on the caudomedial cerebellar surface caused by the BPC. c Postoperative T2-weighted MR image demonstrating the decreased size of both third and fourth ventricle. d Image obtained during postmortem examination demonstrating massive cerebral necrosis and hemorrhagic Blake's pouch cyst with typical mass effect on both caudomedial cerebellar hemispheres
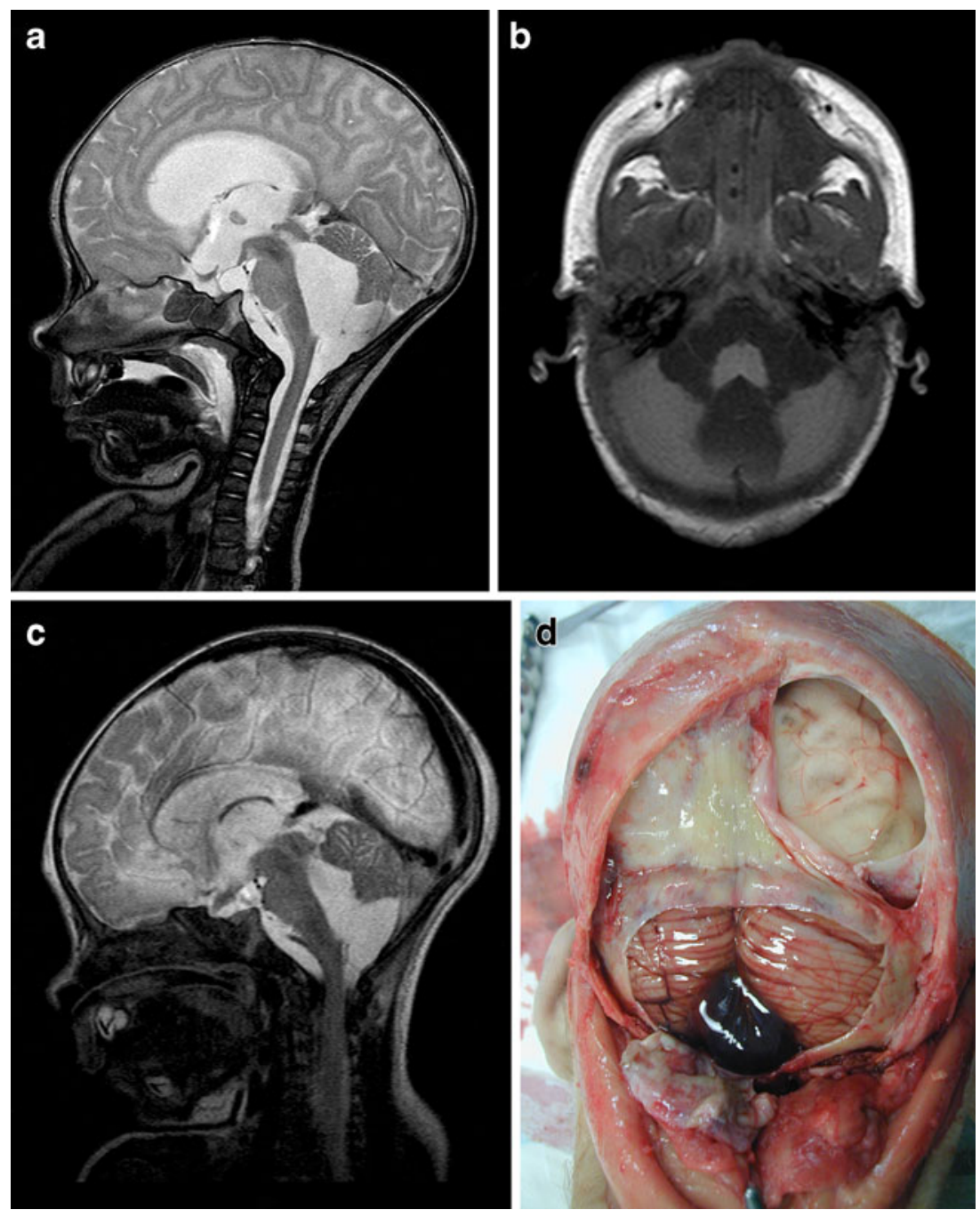
parents. In retrospect, in his second year of life, his head circumference had started to increase reaching a value of $+2.5 \mathrm{SD}(54 \mathrm{~cm})$ at 2 years and 4 months. His language skills were normal. We noted frontal bossing, some congestion of the scalp veins, a normal vertical gaze, and an ataxic gait. MR imaging demonstrated a marked tetraventricular hydrocephalus and a posterior fossa cyst compatible with a BPC (Fig. 2a-c). After ETV, his head circumference stabilized, his gait improved, and he is doing fine with 2 years of follow-up.

\section{Case 3}

At the age of 2 years and 9 months, an otherwise healthy boy presented to our outpatient clinic with a persistent open fontanel. Moreover, his head circumference had increased from P50 at 1 month to P98 at 15 months and $2 \mathrm{~cm}$ above P97 at 2 years and 9 months. We noted frontal bossing, some congestion of the scalp veins, and an open fontanel. MR imaging demonstrated a moderate tetraventricular hydrocephalus and a posterior fossa cyst compatible with a BPC (Fig. 3a-c). Three months after ETV, his head circumference had stabilized and his fontanel had almost closed. He is doing fine with 2.5 years of follow-up.

\section{Case 4}

At the age of 1 year and 10 months, an otherwise healthy girl was admitted with a Listeria meningitis. Despite 5 days of treatment with gentamicin, amoxicillin, and acyclovir, her clinical condition deteriorated with decreased conscious- ness and an abducens paresis. Emergency computed tomography (CT) scan (Fig. 4a) and subsequent MR scan (Fig. 4b) demonstrated marked tetraventricular hydrocephalus, which we interpreted as a communicating (aresorptive) hydrocephalus in the acute phase of a meningitis (Fig. 4c). We inserted an external ventricular drain and surprisingly found a low intraventricular pressure; however, after surgery, the girl's clinical condition rapidly improved. A week later, the drain was clamped for $24 \mathrm{~h}$, which was well tolerated, and subsequently removed. To our surprise, the ventricles on MR scan $24 \mathrm{~h}$ after drain removal were as large as on initial CT scan, whereas the girl was doing fine. At that moment, we noted the BPC and concluded that (a) the hydrocephalus must have been preexistent and associated with her BPC and (b) the hydrocephalus may have temporarily decompensated due to the meningitis. She is doing fine without further treatment with a follow-up of 17 months.

\section{Case 5}

A healthy 69 -year-old man initially noticed slower reactions while playing tennis and during the next year developed a more complex clinical picture. During the course of each day, his gait became slower and unstable. Furthermore, he noticed short memory disturbances, occasional dysphasia, the daily need for an afternoon nap, frequent headaches, nausea and blurred vision by the end of the day, as well as frequent miction overnight. Finally, he suffered three episodes of ophistotonus after a long, strenuous walk. Because the clinical presentation to some extent resembled the normal pressure hydrocephalus triad, the neurologist presented the patient for
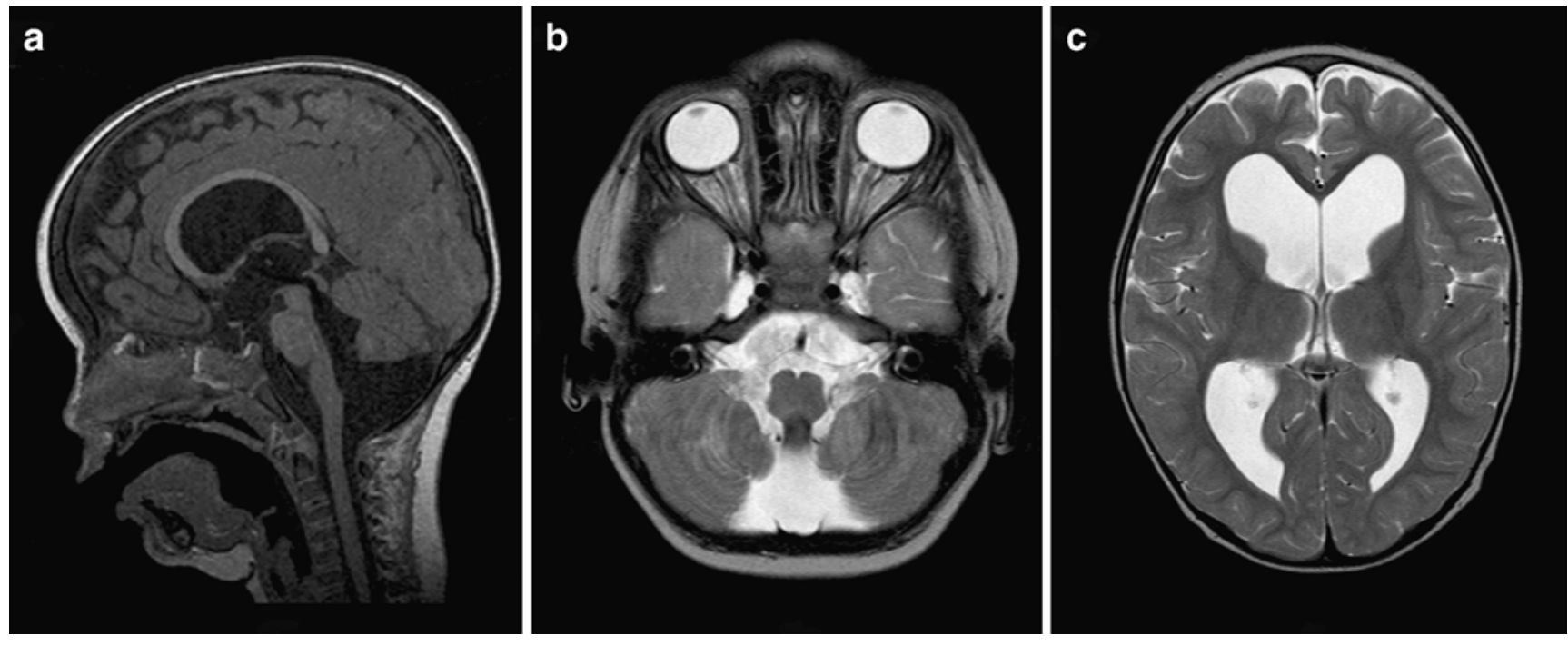

Fig. 2 a Preoperative sagittal T1-weighted MR image demonstrating marked hydrocephalus with bulging third ventricular floor, downward bending mammillary bodies, and an open aqueduct. The cerebellum is not rotated. A thin vertical line between dilated fourth ventricle and cisterna magna indicates a Blake's pouch cyst. The inferior lobules of the vermis are flattened. b Preoperative axial T2-weighted MR image demonstrating a bilateral indentation on the caudomedial cerebellar surface similar to Fig 1b. c Preoperative axial T2-weighted MR image demonstrating enlarged lateral ventricles without periventricular hyperintensities 
Fig. 3 a-c Preoperative sagittal (a) and axial $(\mathbf{b}, \mathbf{c})$ T2-weighted MR images demonstrating moderate tetraventricular hydrocephalus. Note the flow void between fourth ventricle and Blake's pouch cyst. d Axial CT scan 3 days postoperatively (although at different angle) demonstrating decreased size of the frontal horns
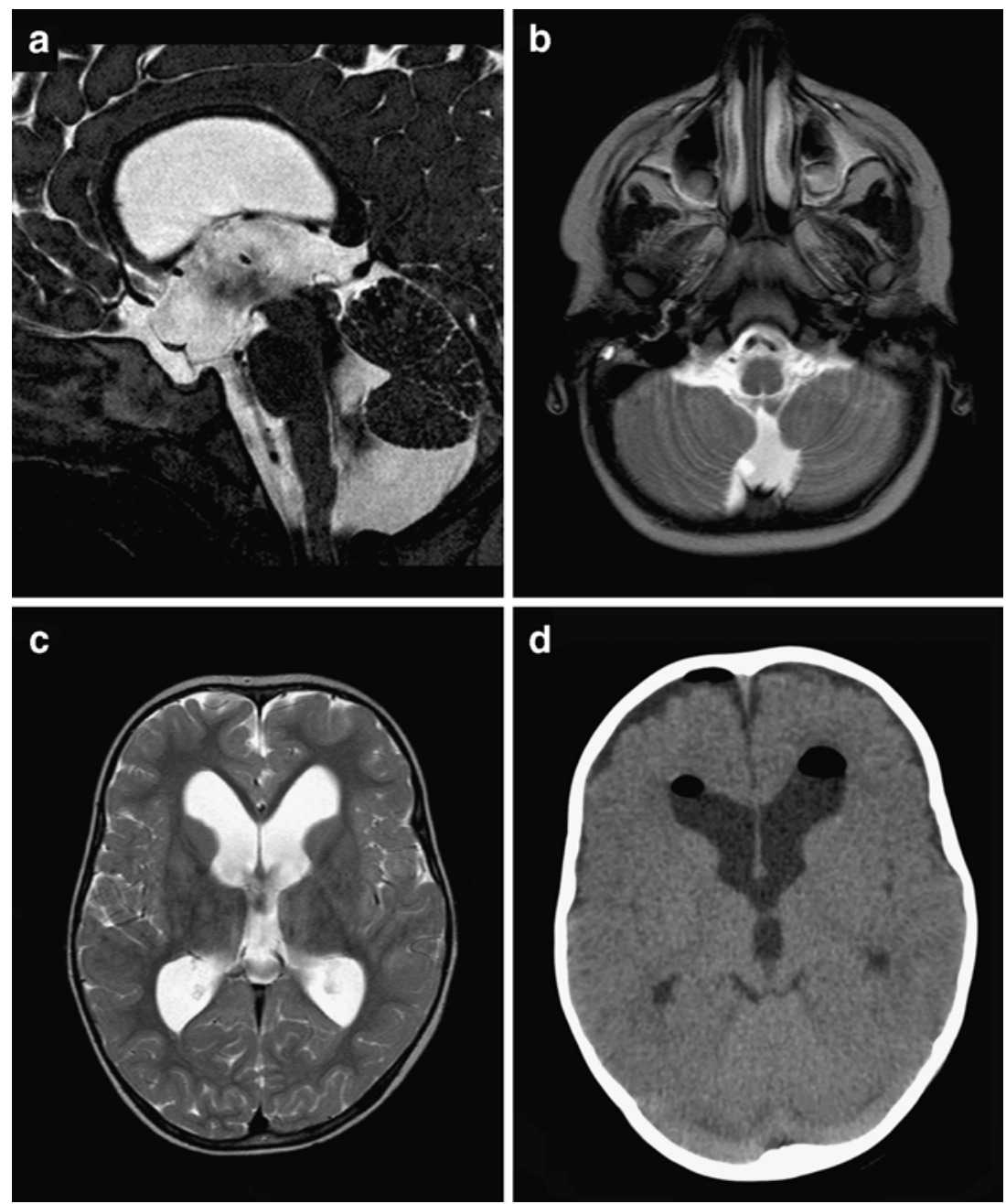
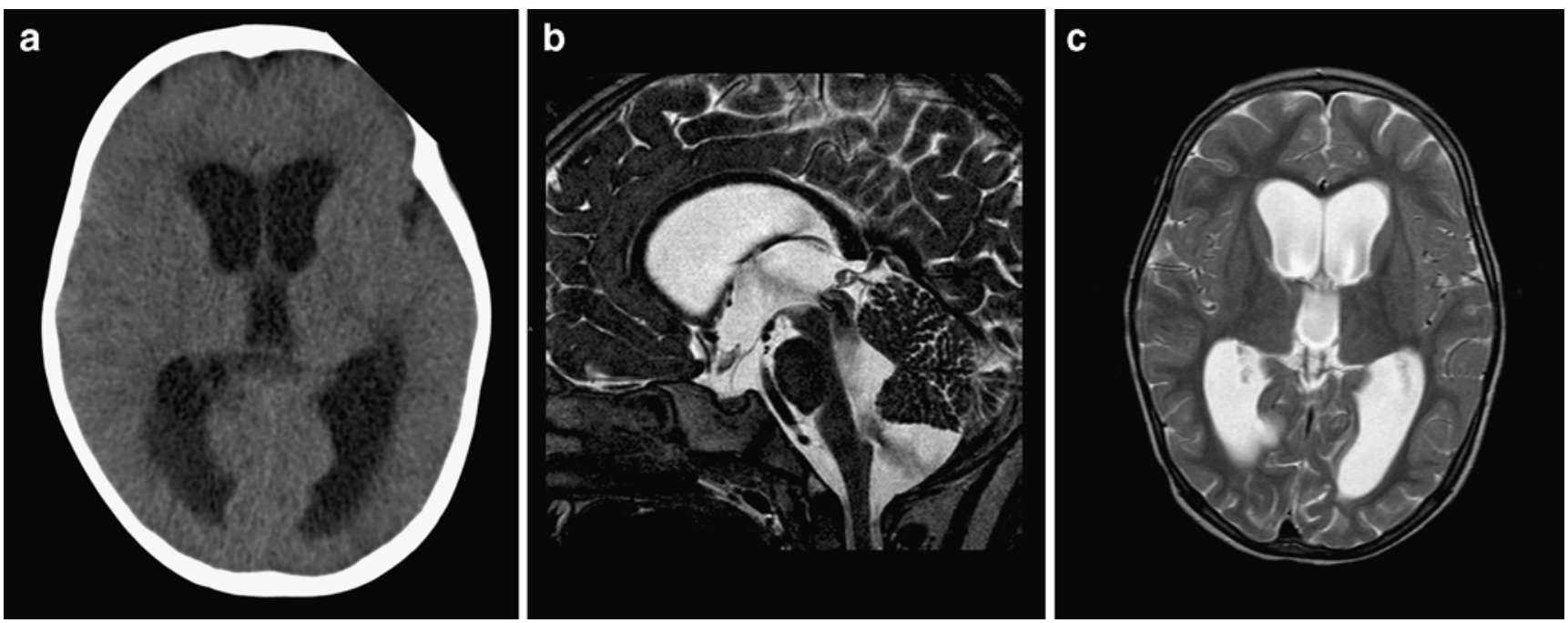

Fig. 4 a Preoperative axial CT scan demonstrating marked hydrocephalus with periventricular hypointensities suggesting increased intraventricular pressure. b, c Postoperative sagittal and axial T2weighted MR images obtained $48 \mathrm{~h}$ after discontinued external ventricular drainage. Ventricular size is identical but periventricular hypointensities are less pronounced as on preoperative images. Note an obvious flow void between fourth ventricle and Blake's pouch cyst (no third ventriculostomy performed) 
evacuating lumbar punctures. Interestingly, we diagnosed a BPC (Fig. 5a-c) indicating an obstructive hydrocephalus and therefore contraindicating evacuating lumbar punctures. Instead, we recently performed an ETV and we are now closely following the patient.

\section{Case 6}

A healthy 51-year-old man presented to the emergency department with ataxia after a bicycle fall. Surprisingly, his CT scan did not reveal any posttraumatic hemorrhage but a moderate tetraventricular hydrocephalus. Two months thereafter, he still complained of minimal concentration difficulties; however, his neuropsychological examination was within normal range. MR imaging was consistent with BPC (Fig. 6a-c) and an apparently arrested (compensated) hydrocephalus. Therefore, no therapy was instituted, and he is doing fine with a follow-up of nearly 2 years.

\section{Discussion}

\section{Embryology of Blake's pouch cyst}

In 1989, using multiplanar MR imaging Barkovich et al. distinguished two basic kinds of posterior fossa cystlike malformations, the DWC, and the arachnoid cyst $[2,3]$. In 1996, Tortori-Donati et al. added another one, BPC, which had not previously been differentiated from megacisterna magna or retrocerebellar arachnoid cyst [11]. BPC was defined as a failure of regression of Blake's pouch (the rudimental fourth ventricular tela choroidea) secondary to nonperforation of the foramen of Magendi, resulting in a posterior ballooning of the superior medullary velum into the cisterna magna [11]. Importantly, during embryological development, the foramina of Luschka open later [8] than the foramen of Magendi [7, 10-12]. Therefore, in case of nonperforation of the foramen of Magendi, the fourth ventricle will enlarge together with the supratentorial ventricles until the foramina of Luschka open and establish a precarious equilibrium of CSF outflow from the ventricles into the cisterns [4]. However, as the larger foramen of Magendi is permanently missing, the ventricles will stay enlarged. Still according to this theory in case of BPC, the cerebellar hemispheres and vermis would rather be compressed (to a certain degree) than underdeveloped and would therefore reexpand in case of ventricular shunting [3]. Finally, most authors agree that BPC and megacisterna magna originate from a defect of the posterior membranous area, whereas DW malformation and DWV originate from a defect of the anterior membranous area $[7,11]$.

Differential diagnosis of Blake's pouch cyst and the importance of MR imaging

Differential diagnosis of BPC includes all other posterior fossa cysts in the DWC, posterior fossa arachnoid cysts, and cystlike malformations. A megacisterna magna is considered to communicate freely with both fourth ventricle and subarachnoid space, whereas a posterior fossa arachnoid cyst represents a CSF collection within duplicated arachnoidal layers which may or may not communicate directly with the subarachnoid space $[4,11]$. Both entities may exert some mass effect on the cerebellum, and may even cause hydrocephalus but less frequently than in BPC and DW malformation. Associated findings such as hypoplasia of the
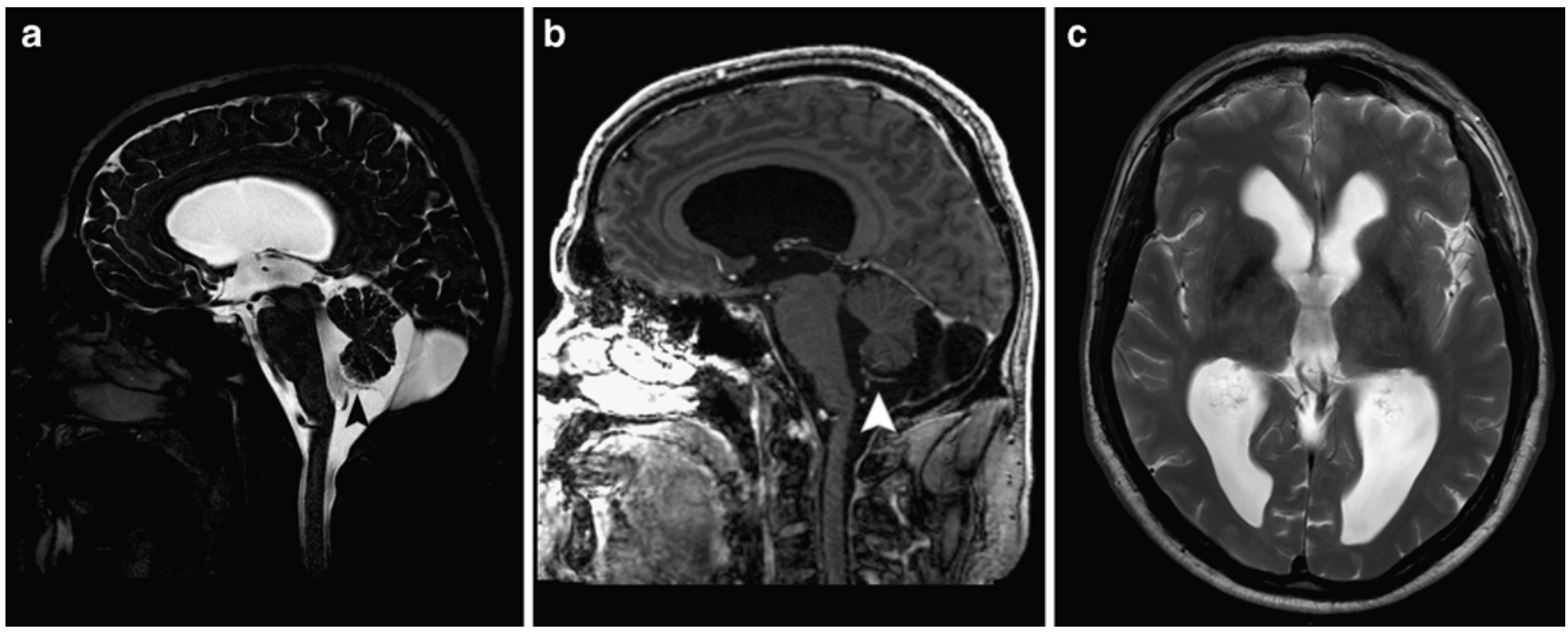

Fig. 5 a-c Preoperative sagittal (a) and axial (c) T2-weighted and postcontrast sagittal T1-weighted (b) MR images demonstrating marked hydrocephalus and continuation of the fourth ventricular plexus into the Blake's pouch cyst (black and white arrowheads). The thin vertical septum behind the cerebellum separates the Blake's pouch cyst from the subarachnoid space 

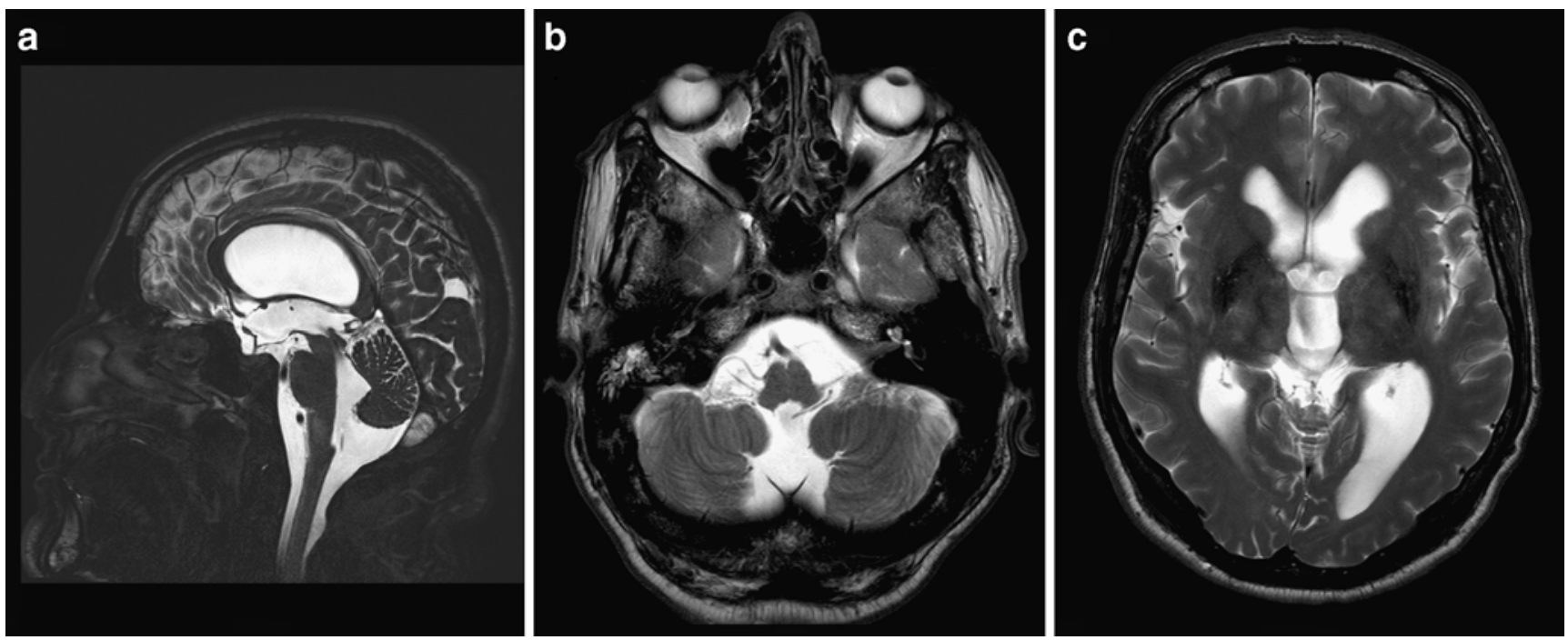

Fig. 6 a-c Sagittal (a) and axial (b, c) T2-weighted MR images demonstrating moderate hydrocephalus with bulging third ventricular floor, downward bending mammillary bodies, and an open aqueduct.

vermis, high position of the torcular, absence of the falx cerebelli, and other central nervous system malformations are typical for a DW malformation and may help to differentiate DW malformation from BPC. Moreover, the position of the fourth ventricular choroid plexus (not to be misinterpreted as a prominent inferior vermian vein) is different in BPC, posterior fossa arachnoid cyst, and DW malformation. After intravenous contrast administration, the choroid plexus is readily seen bending below the vermis and continuing into the pouch in BPC, which is essentially a fourth ventricular diverticulum, whereas it is in a normal position in a posterior fossa arachnoid cyst and nonexistent in DW malformation [7]. Undoubtedly, modern MR imaging has greatly facilitated differential diagnosis and is now indispensable in the diagnostic workup of any retrocerebellar fluid collection [4], allowing assessment of aqueductal and fourth ventricular outlet patency, communication between fourth ventricle, fluid collection, and subarachnoid space, as well as detection of associated central nervous system malformations [4].

The adagium "one only sees what one knows" is certainly true for BPC, as all types of posterior fossa cysts and cystlike malformations may present nearly identical on initial imaging studies [7]. Typical radiological features of BPC are (1) a tetraventricular hydrocephalus, (2) localization of the cyst infra- or retrocerebellar, (3) a relatively well-developed, nonrotated cerebellar vermis (as opposed to a DW), (4) a cystic dilation of the fourth ventricle without cisternal communication, and (5) some degree of compression on the medial cerebellar hemispheres. Ideally, one may see the fourth ventricular choroid plexus continuing in the roof of the cyst on sagittal MR images (as illustrated in Fig. 5a-b).
The cerebellum is not rotated. A thin vertical line between dilated fourth ventricle and cisterna magna indicates a Blake's pouch cyst

Typically (although not invariably), arachnoid cysts and megacisterna magna present without hydrocephalus, which was present in all our cases (Figs. 1, 2, 3, 4, 5, and 6). Finally, the posterior margin of the BPC wall can be visualized on thin sagittal T2-weighted MR images (Figs. 1, 2, 3, 4, 5, and 6), which may help differentiating BPC from megacisterna magna with a coincidental communicating hydrocephalus of unknown etiology (as illustrated in Fig. 4). In line with the theory of Tortori-Donati et al. [11], identification of the fourth ventricular choroid plexus in the roof of the cyst on contrast-enhanced MR images, if present, adds to the diagnosis of BPC; however, in our opinion, routine contrast administration should not be necessary in most cases to make the correct diagnosis.

\section{Clinical presentation of Blake's pouch cyst}

To the best of our knowledge, literature dealing specifically with the clinical presentation and treatment of BPC is nonexistent. Most articles discuss posterior fossa cysts and cystlike malformations altogether, focusing on embryology, classification, imaging, and, to some extent, treatment [1-6, 9-11]. For example, Arai and Sato present a series of 26 patients with posterior fossa cysts, including one patient with a BPC, a 17-month-old-girl presenting with hypotonia and delayed gait development, treated with ventriculoperitoneal shunting, resection of the posterior cyst wall, and cystoperitoneal shunting (as most of their patients) [1]. Calabrò et al. present two middle-aged adults: the first one presenting with syncopal episodes of several minutes duration and the second one with recurrent headaches, vertigo, frequent falls, and mild papilledema [4]. They do 
not elaborate on treatment or follow-up of their patients and merely suggest cyst shunting and ventricular shunting. Conti et al. present a 37-year-old woman with a BPC and a holocord syrinx. She initially improved after decompressive craniectomy and cyst fenestration but rapidly relapsed and was treated with ventriculoperitoneal shunting [5].

To fill this gap, we present six patients with a BPC, including four young children (ranging from 1 month till 2 years and 9 months old at time of diagnosis) and two adults (51 and 69 years old at time of diagnosis). Diagnosis was made on high-quality MR images (all cases) and confirmed by postmortem examination (case 1). Interestingly, all patients had a different clinical presentation, ranging from life-threatening intracranial hypertension at the age of 1 month (case 1) to an incidental CT finding at the age of 51 years (case 6), in line with the observation of Siebert [9] that hydrocephalus in early life associated with posterior fossa anomalies may be asymptomatic until adulthood.

Case 1 was the most severely affected and truly was a unique case, as this 1-month-old boy also had a cholestatic disorder of unknown origin (even after postmortem examination) causing severe coagulopathy. We postulate that this coagulopathy caused a bleeding in a previously asymptomatic BPC, resulting in a peracute high-pressure hydrocephalus with lethal consequences. In this scenario, the BPC was a locus minoris resistentiae, much in analogy with a bleeding temporal arachnoid cyst after a head trauma. Cases 2 and 3 presented between the age of 2 and 3 years. The former one had delayed gait development and an unnoticed pathological increase in head circumference, whereas the latter had normal neurodevelopment, a persistent open fontanel, and a pathological increase in head circumference. Although both boys manifested frontal bossing and congestion of the scalp veins to some degree, neither of them manifested symptoms of intracranial hypertension. Both were successfully treated with ETV, with a follow-up of approximately 2 years. Case 4 presented at the age of 1 year and 10 months with normal neurodevelopment and a Listeria meningitis. We postulate that this meningitis caused a temporary decompensation of a preexistent, compensated hydrocephalus. Indeed, although intraventricular pressure was actually low during external ventricular drain insertion, her clinical condition rapidly improved postoperatively. One week thereafter, the girl was successfully weaned of external ventricular drainage. She is now being closely monitored and is doing well with a follow-up of 17 months so far. Case 5 presented with a rather complex clinical picture at the age of 69 years, to some extent resembling the clinical triad of normal pressure hydrocephalus. Finally, case 6 presented as an asymptomatic 51-year-old adult with an incidental BPC and an apparently arrested (compensated) hydrocephalus on $\mathrm{CT}$ and MR scans, diagnosed after a minor head trauma and without further consequences so far.
Treatment of Blake's pouch cyst

With regard to treatment options, most authors discuss posterior fossa cysts and cystlike malformations altogether $[1,2,5,6,9,10]$. As hydrocephalus in patients with a BPC is caused by nonperforation of the foramen of Magendi [11], at least theoretically, one might consider to marsupialize the cystic fourth ventricle, trying to reconstruct a foramen of Magendi and to reestablish the normal CSF pathway. If successful, the operation might decrease the size of the pouch and allow reexpansion of the cerebellar hemispheres and vermis. According to Tortori-Donati et al., shunting the lateral ventricles (or pouch) restores normal ventricular size, including collapse of the cystic fourth ventricle [11]. Conti et al. observed that cyst fenestration was insufficient to treat an associated holocord syrinx and had to insert a ventriculoperitoneal shunt instead. Interestingly, they concluded that ETV might have been another treatment option [5]. In our experience (as illustrated in cases 2, 3, and 5), ETV is a safe and effective treatment option in patients presenting with a symptomatic BPC, avoiding the risks and added morbidity of open surgery, as well as shunt dependency with obstruction, overdrainage, and other shuntrelated problems well known to pediatric neurosurgeons worldwide. In this regard, ETV may actually be regarded the best current treatment option for symptomatic BPC; however, an analogous discussion about the best treatment option (long-term benefit and cost of CSF shunting versus ETV) in uncomplicated cases of aqueductal stenosis in infants under the age of 24 months is still ongoing, and answers of this International Infant Hydrocephalus Study (Constantini et al., http://147.188.78.52/iihs) are eagerly awaited. It is our hope that the results of this study can, to some extent, be extrapolated to infants diagnosed with symptomatic BPC at an early age.

\section{Conclusion}

Although Blake's pouch cyst is frequently mentioned in the spectrum of posterior fossa cysts and cystlike malformations, its natural history, clinical manifestation, specific imaging characteristics, optimal treatment, and outcome are relatively unknown. Consequently, BPC may still be underdiagnosed. In fact, BPC may present with impaired neurological development and/or progressive hydrocephalus at an early age, may remain asymptomatic throughout life, and may even become symptomatic at an advanced age. Occasionally, other factors such as coagulopathy (causing intracystic bleeding) or meningitis may cause an acute decompensation of a previously compensated hydrocephalus. Differentiating BPC from other posterior fossa cysts and cystlike malformations and recognizing the accompanying 
hydrocephalus are essentially noncommunicating, not only have important implications on clinical management but also on genetic counseling (unnecessary in case of BPC). High-quality MR imaging is indispensable in the diagnostic workup of any retrocerebellar fluid collection and has greatly facilitated differential diagnosis, with some features pointing specifically to a BPC. In our experience, ETV is a safe and effective treatment option in patients presenting with a symptomatic BPC, avoiding the risks and added morbidity of open surgery, as well as many shunt-related problems. However, before ETV may be regarded the best treatment option, larger, prospectively randomized studies including thorough neuropsychological follow-up are needed.

Open Access This article is distributed under the terms of the Creative Commons Attribution Noncommercial License which permits any noncommercial use, distribution, and reproduction in any medium, provided the original author(s) and source are credited.

\section{References}

1. Arai H, Sato K (1990) Posterior fossa cysts: clinical, neuroradiological and surgical features. Child's Nerv Syst 7:156-164

2. Barkovich AJ, Kjos BO, Norman D, Edwards MS (1989) Revised classification of posterior fossa cysts and cystlike malformations based on the results of multiplanar MR imaging. AJNR 10:977988

3. Barkovich AJ (1994) Pediatric neuroimaging, 2nd edn. Raven, New York, pp 177-275

4. Calabrò F, Arcuri T, Jinkins JR (2000) Blake's pouch cyst: an entity within the Dandy-Walker continuum. Neuroradiology 42:290-295

5. Conti C, Lunardi P, Bozzao A, Liccardo G, Fraioli B (2003) Syringomyelia associated with hydrocephalus and Blake's pouch cyst: case report. Spine 28(14):E279-E283

6. Cinalli G, Spennato P, del Basso de Caro ML, Buonocore MC (2004) Hydrocephalus and the Dandy-Walker malformation. In: Cinalli G (ed) Pediatric hydrocephalus. Springer, Milan, pp 259277

7. Nelson MD Jr, Maher K, Gilles FH (2004) A different approach to cysts of the posterior fossa. Pediatric Radiol 34:720-732

8. Raybaud C (1982) Les malformations kystiques de la fosse postérieure. J Neuroradiol 9:103-133

9. Siebert JR (2006) A pathological approach to anomalies of the posterior fossa. Birth defects research (part A): clinical and molecular. Teratology 76:674-684

10. Strand RD, Barnes PD, Young Poussaint T, Estroff A, Burrows PE (1993) Cystic retrocerebellar malformations: unification of the Dandy-Walker complex and the Blake's pouch cyst. Pediatr Radiol 23:258-260

11. Tortori-Donati P, Fondelli MP, Rossi A, Carini S (1996) Cystic malformations of the posterior cranial fossa originating from a defect of the posterior membranous area, mega cisterna magna and persisting Blake's pouch: two separate entities. Child's Nerv Syst 12:303-308

12. Zalel Y, Gilboa Y, Gabis L, Ben-Sira L, Hoffman C, Wiener Y, Achiron R (2006) Rotation of the vermis as a cause of enlarged cisterna magna on prenatal imaging. Ultrasound Obstet Gynecol $27: 490-493$ 